\title{
MicroRNA-518d regulates PPAR $\alpha$ protein expression in the placentas of females with gestational diabetes mellitus
}

\author{
CHUN ZHAO $^{1 *}$, TING ZHANG ${ }^{2 *}$, ZHONGHUA SHI $^{1}$, HONGJUAN DING $^{1}$ and XIUFENG LING ${ }^{1}$ \\ ${ }^{1}$ State Key Laboratory of Reproductive Medicine, Nanjing Maternity and Child Health Care Hospital \\ Affiliated to Nanjing Medical University, Nanjing, Jiangsu 210004; ${ }^{2}$ Wuxi Maternity and Child Health \\ Hospital Affiliated to Nanjing Medical University, Wuxi, Jiangsu 214002, P.R. China
}

Received August 15, 2013; Accepted March 3, 2014

DOI: $10.3892 / \mathrm{mmr} .2014 .2058$

\begin{abstract}
The placenta is thought to have a critical role in the pathogenesis of gestational diabetes mellitus (GDM), as GDM-associated complications resolve following delivery. Placenta-specific microRNAs (miRNAs) may contribute to the pathology of the development of GDM. The aim of the present study was to evaluate whether the placenta-specific miR-518d contributes to the development of GDM. It was revealed that miR-518d expression was higher in placentas taken from patients with GDM compared with control placentas, whereas the protein levels of the predicted miR-518d target gene, peroxisome proliferator-activated receptor- $\alpha$ (PPAR $\alpha)$, were lower in placentas from patients with GDM compared with those from control subjects. It was also demonstrated that PPAR $\alpha$ was a direct target of miR-518d with a specific binding site at the seed sequence, which determines target specificity. In the placentas of females with GDM increased levels of miR-518d were negatively correlated with the levels of PPAR $\alpha$ protein. As PPAR $\alpha$ dysregulation may be related to the development of GDM, it is suggested that upregulation of miR-518d may be associated with the pathogenesis of GDM via an effect on the regulation of PPAR $\alpha$ expression.
\end{abstract}

\section{Introduction}

Gestational diabetes mellitus (GDM), characterized by glucose intolerance diagnosed during pregnancy, is one of

Correspondence to: Professor Xiufeng Ling, State Key Laboratory of Reproductive Medicine, Nanjing Maternity and Child Health Care Hospital Affiliated to Nanjing Medical University, Nanjing Medical University, Nanjing, Jiangsu 210004, P.R. China

E-mail: lingxiufeng_njfy@163.com

*Contributed equally

Abbreviations: GDM, gestational diabetes mellitus; PPAR $\alpha$, peroxisome proliferator-activated receptor-alpha; miR-SCR, miR-scrambled control; OGTT, oral glucose tolerance test; pRL-TK, renilla luciferase reporter vector

Key words: gestational diabetes, miR-518d, micro RNA, peroxisome proliferator-activated receptor- $\alpha$, placenta the most common complications in pregnancy and affects $3-8 \%$ of all pregnancies $(1,2)$. The prevalence of GDM has increased in recent decades due to increased average age of pregnant females and increased risk of obesity (3). However, GDM is associated with numerous complications including macrosomia, neonatal metabolic disorders, respiratory distress syndrome and neonatal death as well as a predisposition for the development of metabolic syndromes and type 2 diabetes $(4,5)$.

The mammalian placenta serves as an auxiliary fetal organ at the interface between the mother and the fetus. It is a vitally important endocrine organ during pregnancy. In addition to the production of a wide variety of steroids, peptides and further regulatory factors, the placenta is an endocrine target tissue, expressing a broad spectrum of hormone receptors and growth factor receptors (6). Growth and differentiation of the placenta are fundamental to mammalian reproduction, and functional impairment of this organ occasionally leads to severely abnormal pregnancies (7). The aberrant development and function of the placenta have been suggested as important contributory factors to GDM-associated complications (8) and, as the condition is resolved following delivery, it is likely that the placenta has a critical role in the pathogenesis of GDM (10).

Studies have revealed that microRNA (miRNA) expression is tissue-specific and it has been reported that some miRNAs are specific to, and highly expressed in, the placenta (placental-specific miRNAs) $(8,9)$. In a recent study, it was shown that certain abnormal pregnancies are associated with alterations in miRNA expression in the placenta (9). Two large miRNA clusters expressed in placenta have been described: C19 MC (located at chromosome 19q13.41), which comprises 54 predicted miRNAs, and C14 MC (located at chromosome 14q32), which contains 46 miRNAs (8). It has been reported that these placenta-specific miRNAs may contribute to the pathology of abnormal pregnancies, including pre-eclampsia and intrauterine growth restriction (9). However, few reports have described the involvement of miRNAs in the regulation of the development of GDM.

miR-518d is one of the miRNAs from the C19 MC cluster and is highly expressed in the placenta. In a previous study, our group showed that the miR-518d microRNA was differentially expressed in placentas from patients with GDM. 
Bioinformatic analysis has predicted the peroxisome proliferator-activated receptor- $\alpha$ (PPAR $\alpha)$ to be a target for miR-518d. The peroxisome proliferator-activated receptors (PPARs) are a family of fatty acid receptors that transduce stimuli from fatty acids into alterations in gene expression. PPARs have a critical role in lipid homeostasis and inflammation, and have long been linked to the diabetic phenotype (11). During pregnancy, dynamic physiological, metabolical and immunological adaptations are required to ensure fetal development and maternal well-being. A large number of the metabolic adaptations are mediated by PPARs (12). Dysregulation of PPARs may result in GDM, as it has been reported that the expression levels of the PPAR $\alpha$ protein and mRNA are lower in placentas from females with GDM compared with controls without GDM (13). The present study investigated whether miR-518d is involved in the development of GDM and whether PPAR $\alpha$ expression is suppressed by miR-518d in placentas from females with GDM.

\section{Materials and methods}

Patients and tissue samples. Human placentas were obtained according to protocols approved by the Ethics Committee of Nanjing Maternity and Child Healthcare Hospital, affiliated to Nanjing Medical University. All the patients provided written informed consent before taking part in the study. Full-term placental tissue samples were obtained from elective terminations of pregnancy and uncomplicated cesarean deliveries, respectively. Placentas were obtained from females who delivered from 37-40 weeks of gestation. Gestational age, recorded as completed weeks of gestation, was calculated from the date of the last menstrual period and/or from ultrasound. Females were diagnosed with GDM when a $75 \mathrm{~g}$ oral glucose tolerance test (OGTT) revealed either a fasting venous plasma glucose level $>5.6 \mathrm{mmol} / 1$ glucose and/or a $2 \mathrm{~h}$ post-test plasma glucose level $>8.6 \mathrm{mmol} / \mathrm{l}$ glucose. None of the patients had a previous history of diabetes mellitus or any known endocrinopathy. Placental tissue was collected at delivery and immediately transferred on ice for transport to the laboratory for RNA isolation, protein lysate preparation and tissue fixation.

Quantitative polymerase chain reaction ( $q P C R$ ). Total RNA was extracted from placentas with TRIzol reagent (Invitrogen Life Technologies, Carlsbad, CA, USA). miR-518d was reverse transcribed into complementary DNA (cDNA) using a TaqMan microRNA assay (Applied Biosystems, Branchburg, NJ, USA) containing microRNA-specific stem-loop RT primers and a TaqMan MicroRNA Reverse Transcription kit in a total reaction volume of $50 \mu \mathrm{l}$, performed according to the manufacturer's instructions.

Reverse transcriptase reactions were performed using a 7300 real-time PCR system (Applied Biosystems, Beijing, China) and the following thermal cycling parameters: $30 \mathrm{~min}$ at $16^{\circ} \mathrm{C}, 30 \mathrm{~min}$ at $42^{\circ} \mathrm{C}, 5 \mathrm{~min}$ at $85^{\circ} \mathrm{C}$ and then held at $4^{\circ} \mathrm{C}$. miRNA expression was normalized to the expression level of small nucleolar RNA U6. The primer of miR-518d was F: 5'-ACACTCCAGCTGGGCAAAGCGCTTCCCTT-3' and R: 5'-CTCAACTGGTGTCGTGGAGTCGGCAATTCAGTT GAGCTCCAAA-3'.
Luciferase reporter expression assays using luciferase genes with mutated or wild-type PPAR $\alpha$-3'-untranslated region (UTR) in human embryonic kidney (HEK) cells co-transfected with miR-518d or microRNA-scrambled control (miR-SCR). Wild-type PPAR $\alpha-3$ '-UTR was amplified by PCR from human cDNA using the primers: F: 5'-CCA AGCTTCGTCCAGTCAACCTGAACCCA-3' and R: 5'-CGA GCTCCTCCAGGTGCCCAGCGACT-3'. The mutated PPAR $\alpha-3$ '-UTR was amplified using the primers: F: 5'-CCA AGCTTCGTCCAGTCAACCTGAACCCA-3' and R: 5'-CGA GCTCCACGACGTGCCCAGCGACT-3'. The DNA segments produced from these amplifications were inserted into the pMIR-REPORT miRNA Expression Reporter Vector (Ambion, Carlsbad, CA, USA) using the SacI and HindIII sites. HEK-293 cells cultured in 24-well plates were co-transfected with pMIR-REPORT vectors containing either the wild-type or mutated PPAR $\alpha-3$ '-UTR segments along with the control vector, renilla luciferase reporter vector (pRL-TK). These HEK-293 cells were co-transfected with the precursor microRNAs for miR-518d (pre-miR-518d) and miR-SCR. Assays were performed to determine the level of gene expression $48 \mathrm{~h}$ post-transfection using the Dual Luciferase Reporter Assay kit (Promega, Madison, WI, USA). Renilla luciferase activity was used to normalize the luciferase activity. Three independent experiments were performed in triplicate.

Immunohistochemistry. Formalin-fixed placental tissue samples were embedded in paraffin, sectioned at $5 \mu \mathrm{m}$, and mounted on silane-coated slides. The sections were dewaxed and rehydrated through descending grades of alcohol to distilled water, followed by blocking of endogenous peroxidase activity using 3\% (v/v) hydrogen peroxidase in phosphate-buffered saline (PBS). The sections were subjected to microwave antigen retrieval in $0.02 \mathrm{M}$ ethylenediaminetetraacetic acid (EDTA), washed in PBS and blocked with goat serum (Beijing ZhongShan Biotechnology, Beijing, China) for $2 \mathrm{~h}$, then incubated overnight at $4^{\circ} \mathrm{C}$ with polyclonal anti-PPAR $\alpha$ (1:200, Abcam, Cambridge, MA, USA). Following three washes in PBS, the sections were incubated with horseradish peroxidase (HRP)-conjugated secondary antibody $(1: 1,000$; Beijing ZhongShan Biotechnology, Beijing, China) for $1 \mathrm{~h}$ at room temperature. Immunoreactivity was demonstrated using diaminobenzidine (Sigma, St. Louis, MO, USA) for increased sensitivity, which produces a brown precipitate at immunopositive sites. Sections were counterstained with hematoxylin and mounted with a coverglass. The negative controls were incubated with immunoglobulin $\mathrm{G}$ ( $\mathrm{IgG}$ ) controls. All the immunostained sections were evaluated in a blinded manner by two observers.

Protein extraction and western blot analysis. For western blot analysis, nuclear proteins from $200 \mathrm{mg}$ of frozen placenta were extracted. Protein lysates were prepared in the presence of protease inhibitors $[10 \mu \mathrm{g} / \mathrm{ml}$ aprotinin, $5 \mu \mathrm{g} / \mathrm{ml}$ leupeptin, $1 \mathrm{mM}$ 4-(2-aminoethyl)benzenesulfonyl fluoride (AEBSF), $1 \mathrm{mM} \mathrm{Na} \mathrm{VO}_{4}$ and $1 \mathrm{mM} \mathrm{NaF}$. Protein concentrations were determined using the Bradford protein assay. Samples containing $50 \mu \mathrm{g}$ of protein were subjected to electrophoresis on a $12 \%$ SDS-PAGE and transferred to a nitrocellulose membrane (GE Healthcare, San Francisco, CA, USA). The 
Table I. Clinical characteristics of females with GDM and controls.

\begin{tabular}{lccc}
\hline Variables & Control $(\mathrm{n}=40)$ & GDM $(\mathrm{n}=40)$ & $\mathrm{P}$-value \\
\hline Maternal age (years) & $30.23 \pm 3.02$ & $30.55 \pm 3.30$ & $\mathrm{NS}$ \\
Gestational age at delivery (week) & $39.64 \pm 0.92$ & $39.61 \pm 0.76$ & $\mathrm{NS}$ \\
Body mass index $\left(\mathrm{kg} / \mathrm{m}^{2}\right)$ & $21.87 \pm 2.56$ & $23.54 \pm 3.46$ & 0.023 \\
Neonatal birth weight $(\mathrm{kg})$ & $3.572 \pm 0.306$ & $4.007 \pm 0.489$ & 0.004 \\
Smokers during pregnancy & 0 & 0 & $\mathrm{NS}$ \\
Placental weight $(\mathrm{kg})$ & $551 \pm 121$ & $572 \pm 132$ & $\mathrm{NS}$
\end{tabular}

GDM, gestational diabetes mellitus; NS, not significant, i.e., $\mathrm{P}>0.05$.

membranes were blocked in Tris-buffered saline (TBS) containing 5\% non-fat milk powder for $1 \mathrm{~h}$ and then incubated overnight with the polyclonal antibodies anti-PPAR $\alpha$ (1:500, Abcam, Cambridge, MA, USA) and anti-GAPDH (1:1,000, Kangcheng, Shanghai, China) diluted in a solution of $5 \%$ non-fat milk powder in TBS. GAPDH was used as a control. Membranes were washed three times with TBS (10 min each) and then incubated for $1 \mathrm{~h}$ with HRP-conjugated goat anti-rabbit IgG (1:1,000; Beijing ZhongShan Biotechnology). Specific proteins were detected using a chemiluminescence ECL kit and AlphaImager (FluorChem5500, Alpha Innotech, San Leandro, CA, USA). Protein expression levels were analyzed using AlphaEaseFC software (Alpha Innotech, San Leandro, CA, USA).

Statistical analysis. Data from at least three independent experiments were expressed as the mean \pm standard deviation (SD). The differences between groups were analyzed using the Student's t-test. The correlation between the relative expression levels of miR-518d and the expression levels of PPAR $\alpha$ was analyzed using a two-sided Spearman's Q-test. Differences between data were considered significant if $\mathrm{P}<0.05$.

\section{Results}

Clinical characteristics of the patients. Table I shows the clinical characteristics of the patients included in the present study and compares these characteristics in females with GDM $(n=40)$ with those of females in the control group $(n=40)$. No significant differences were identified between the two groups with respect to maternal age, gravida, parity or number of weeks of gestation at delivery. Females with GDM had a significantly greater mean body mass index (BMI) and the neonates had a significantly higher birth weight than those whose mothers were in the control group.

Aberrant expression of miR-518d in GDM placenta. To investigate whether miR-518d is involved in the development of GDM, PCR analysis was used to study the expression levels of miR-518d in placental tissue from patients with GDM and normal controls. The expression levels of miR-518d were significantly higher in the placenta of patients with GDM than those in the placentas of the normal controls (Fig. 1). The results suggest that miR-518d may be associated with the development of GDM.

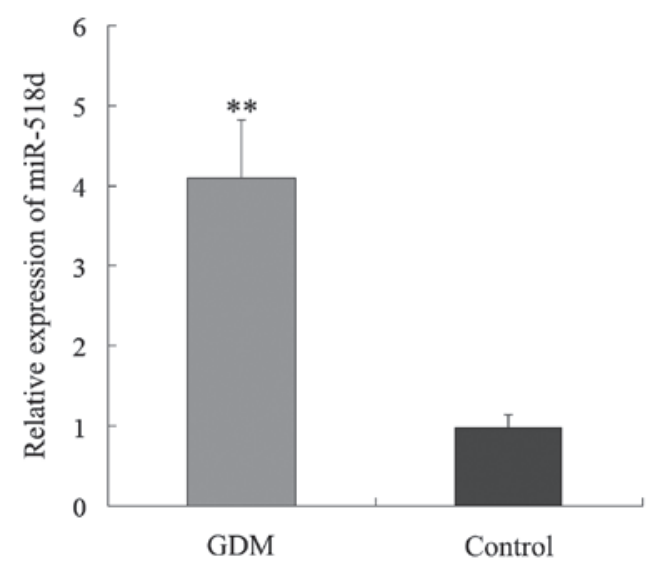

Figure 1. As quantified by PCR, miR-518d levels in placentas from females with GDM were significantly increased compared with those of healthy pregnant females. U6 RNA levels were used as an internal control. ${ }^{* *} \mathrm{P}<0.01$. PCR, polymerase chain reaction; GDM, gestational diabetes mellitus; miR-518d, microRNA-518d.

PPAR $\alpha$ is a direct target of $m i R-518 d$. To identify the potential mechanism by which miR-518d is associated with GDM, three bioinformatic algorithms (TargetScan, PicTar and miRanda) were applied to identify potential target genes for miR-518d. Among the potential candidates, the study focused on the PPAR $\alpha$ gene as PPARs are connected with the diabetic phenotype (11). One miR-518d-binding site was found in the 3'-UTR of PPAR $\alpha$ mRNA (Fig. 2A). However, there are no reports as yet describing PPAR $\alpha$ regulation by miRNA-518d.

Expression of luciferase gene with mutated or wild-type PPAR $\alpha-3$ '-UTR in HEK cells co-transfected with miR-518d or $m i R-S C R$. Expression assays were performed with the luciferase reporter gene system using the wild-type PPAR $\alpha-3$ '-UTR or a mutated version to validate the miR-518d target prediction. The vector was constructed by inserting the wild-type sequence of the PPAR $\alpha$ 3'-UTR PPAR $\alpha$ mRNA (PPAR $\alpha-3$ '-UTR) or a mutated seed sequence of the miR-518d-binding site (PPAR $\alpha$-3'-UTR-mut) into the 3'-UTR of the pMIR-REPORT luciferase reporter. Co-transfection of the vector with the wild-type PPAR $\alpha-3$ '-UTR and the miR-518d precursor, pre-miR-518d, inhibited luciferase activity, whereas co-transfection of the vector with PPAR $\alpha-3$ '-UTR-mut and pre-miR-518d caused no inhibition of luciferase activity (Fig. 2B). These results validated the hypothesis that miR-518d is able to bind to 
$\mathbf{A}$
PPARa $5^{\prime} \mathrm{C}$
C GGG A
G 3'
CAGGGAGU GCU C C CUGGAG
GUCUUUCA CGA G G GAUCUC
miR-518d 3' A G A 5 ,

B

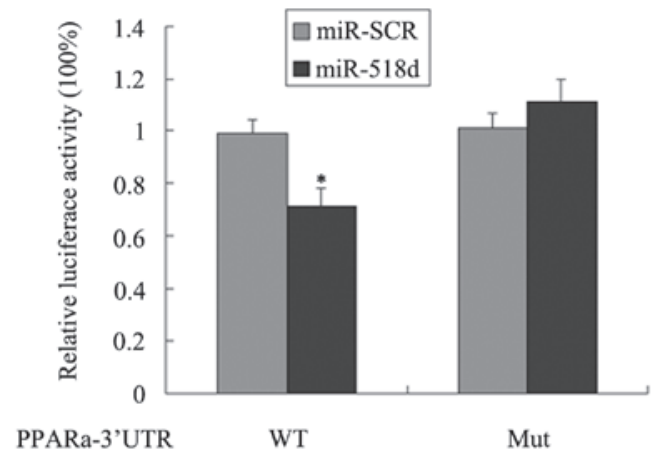

C

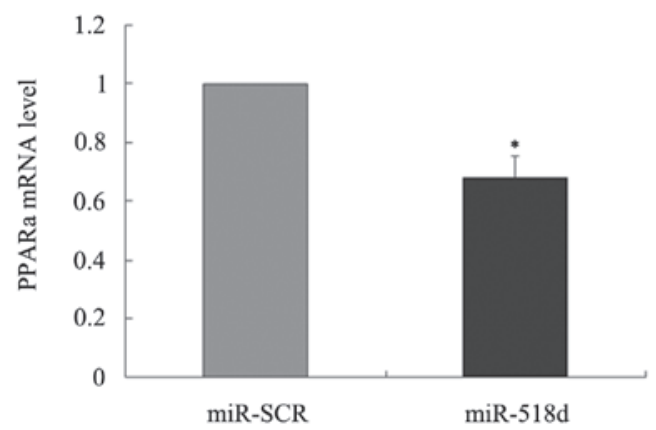

Figure 2. miR-518d directly targets PPAR $\alpha$. (A) Schematic diagram of the miR-518d binding site in the 3'-UTR of the PPAR $\alpha$ gene. (B) Relative luciferase expression levels obtained from pMIR-REPORT miRNA expression vectors containing wild-type (WT) or mutant (Mut) PPAR $\alpha$ 3'-UTR regions following co-transfection with precursors of miR-SCR or miR-518d, and the Renilla control vector were calculated as the ratio of firefly/renilla luciferase activities in the cells and normalized to those of the control. The results are presented as the mean \pm standard deviation from three independent experiments with each experiment performed in triplicate. (C) Overexpression of miR-518d inhibited PPAR $\alpha$ mRNA expression. ${ }^{*} \mathrm{P}<0.05$. PPAR $\alpha$, peroxisome proliferator-activated receptor- $\alpha$; miR, microRNA; SCR, scrambled control; URT, untranslated region.

the 3'-UTR of PPAR $\alpha$ mRNA. The impact of miR-518d on the expression of PPAR $\alpha$ was also investigated. qPCR revealed that PPAR $\alpha$ mRNA levels decreased significantly $48 \mathrm{~h}$ following transfection of HEK-293 cells with pre-miR-518d (Fig. 2C).

Expression and subcellular location of PPAR $\alpha$ in the placenta of patients with GDM. Immunohistochemical staining showed that PPAR $\alpha$ was located in the nuclei of the syncytiotrophoblasts, and its expression was reduced in placentas of patients with GDM than in the control placentas (Fig. 3A). Western blot analysis was performed to confirm the differential expression of PPAR $\alpha$ in placentas of patients with GDM and the control placentas. The expression levels of PPAR $\alpha$ were significantly reduced in placentas from patients with GDM than those in the controls (Fig. 3B and C).

The correlation between PPAR a and miR-518d in females with GDM. The potential correlation between the levels of

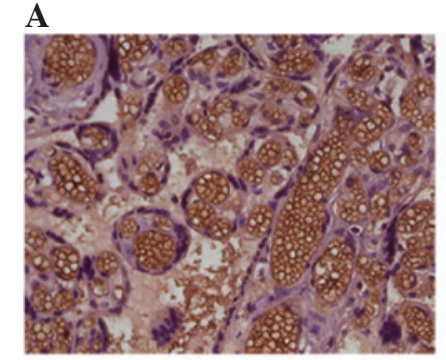

GDM

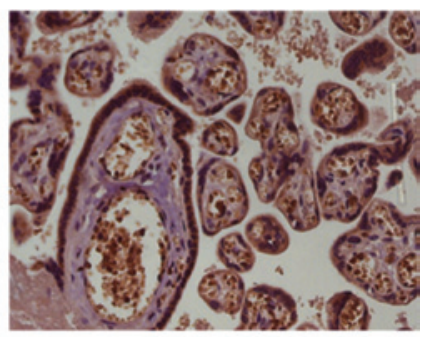

Control
B

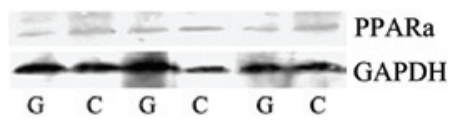

C

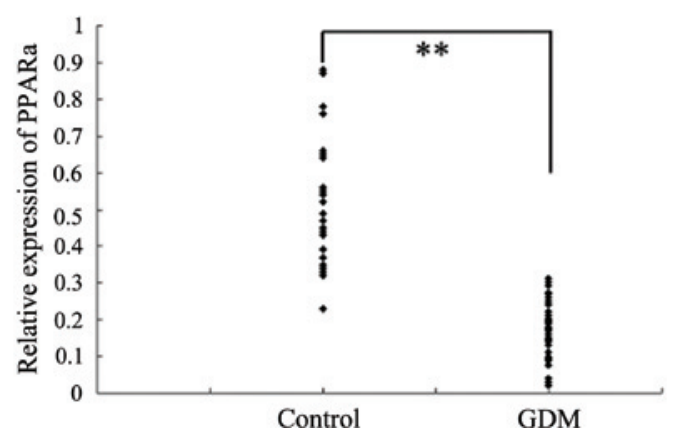

Figure 3. (A) Representative images of immunohistochemical staining for PPAR $\alpha$ protein in the placental tissues of patients with GDM and controls. The nuclear staining of syncytiotrophoblastic and cytotrophoblastic cells was less intense in the placental tissue of patients with GDM compared with that of the controls. (B) Western blot analysis with anti-PPAR $\alpha$ and anti-GAPDH polyclonal antibodies was performed on aliquots of total placental protein from 40 patients with GDM and 40 controls. (C) Comparison of PPAR $\alpha$ protein expression levels in placentas from patients with GDM and controls. The Y-axis represents the relative quantification of $\operatorname{PPAR} \alpha$ normalized to GAPDH. ${ }^{* *} \mathrm{P}<0.01$. PPAR $\alpha$, peroxisome proliferator-activated receptor- $\alpha$; GDM, gestational diabetes mellitus.

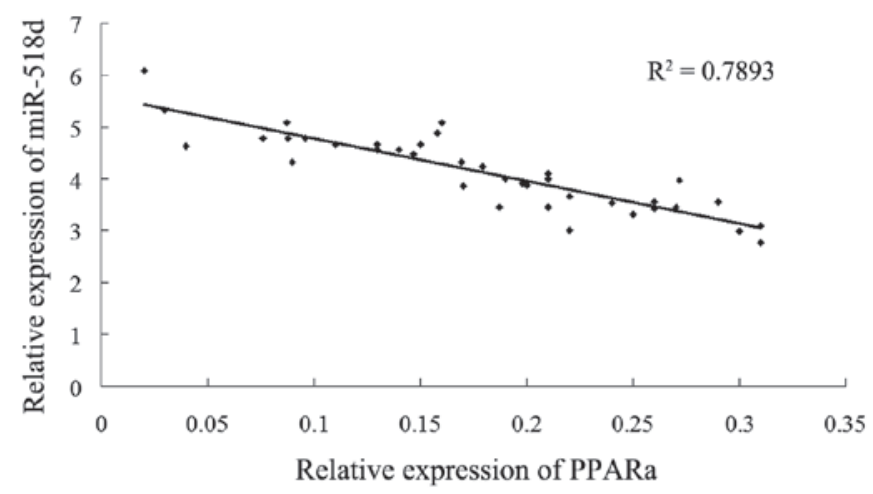

Figure 4. PPAR $\alpha$ protein levels were negatively correlated with miR-518d levels in placentas from patients with GDM, using Spearman's correlation analysis $\left(\mathrm{R}^{2}=0.7893 ; \mathrm{P}<0.01\right)$. miR, microRNA; PPAR $\alpha$, peroxisome proliferator-activated receptor- $\alpha$; GDM, gestational diabetes mellitus.

miRNA-518d expression and PPAR $\alpha$ protein levels in the placentas of females with GDM was assessed using Spearman's correlation analysis. The levels of miR-518d were negatively correlated with the protein levels of PPAR $\alpha$ in females with GDM (Fig. 4; $\mathrm{R}^{2}=0.7893, \mathrm{P}<0.01$ ). 


\section{Discussion}

Recent data indicate that microRNAs have a fundamental role in a variety of physiological and pathological processes. miRNA analyses indicate that diverse affected tissue types have miRNA expression profiles that are significantly different from normal tissue (9). Studies of microRNA expression revealed that some microRNAs are abundantly expressed in the placenta (9). However, the signature of miRNAs in the placenta has yet to be elucidated. Recently, analyses of the expression of small RNAs in the placenta by small RNA library sequencing confirmed that most placenta-specific miRNAs were linked to the $\mathrm{C} 19 \mathrm{MC}$ cluster and some of them have been reported to be associated with pre-eclampsia or premature labor (14). However, the possible involvement of the C19 MC cluster in GDM remains to be elucidated.

MiR-518d is a member of the C19 MC cluster. In a microarray analysis previously performed by our group, the microRNA expression profiles in placentas of patients with GDM were compared with those of normal placentas, which revealed that miR-518d was differentially expressed in placentas of patients with GDM. miR-518d has been reported to be upregulated $>10$-fold in third trimester trophoblast cells compared with first trimester trophoblast cells, indicating that miR-518d may be involved in the regulation of trophoblast proliferation and invasion (14). However, the involvement of placental miR-518d in the molecular mechanisms of GDM requires to be elucidated.

In the present study, expression levels of miR-518d in the placentas of females with GDM were compared with those of normal pregnant females. miR-518d was aberrantly upregulated in placentas of females with GDM compared with controls. This indicates that miR-518d may be associated with the pathogenesis of GDM. The PPAR $\alpha$ gene was selected as a potential target for miR-518d as PPAR $\alpha$ is highly expressed in tissues that catabolize fatty acids. It is a transcription factor that controls fatty acid uptake and metabolism, and it upregulates fatty acid $\beta$ oxidation in these tissues $(16,17)$. It has been reported that regulation of PPAR $\alpha$ expression and activity contributes to maintaining a homeostatic balance between cellular fatty acid and glucose utilization via activation of its target genes (11). Accordingly, activation of PPAR $\alpha$ increases sensitivity to insulin as well as thrombosis and vascular inflammation (18-20). By contrast, it appears that inhibition of PPAR $\alpha$ suppresses sensitivity to insulin and increases hepatic glucose production. GDM is a common complication of pregnancy. GDM is able to lead to dyslipidemia, and is aggravated by obesity. Placental cholesterol and fatty acid transfer have critical roles in the development of GDM (21), and it is possible that the regulation of placental PPAR $\alpha$ may be involved in this process. Consistent with previous studies, the present study shows that the expression levels of PPAR $\alpha$ protein were significantly reduced in the placentas of females with GDM (15). As the level of miR-518d is upregulated and the level of PPAR $\alpha$ is downregulated in placentas of females with GDM, it is hypothesized that miR-518d is involved in the pathophysiology of GDM via its effect on its target gene PPAR $\alpha$. Using the luciferase reporter gene system and mutation assays, the present study confirmed that miR-518d is able to target PPAR $\alpha$ directly by binding to the 3'-UTR of
PPAR $\alpha$ mRNA. In addition, it was revealed that miR-518d levels were negatively correlated with the levels of PPAR $\alpha$ protein in placentas from females with GDM.

In conclusion, the present study has provided evidence that miR-518d has an important role in the pathophysiology of GDM via an inhibitory effect on the expression of PPAR $\alpha$, which may disrupt the balance of fatty acid uptake and metabolism and result in an increased resistance to insulin. Elucidation of this mechanism may offer opportunities for application of miR-518d in future clinical management of females with GDM.

\section{Acknowledgements}

The present study was financially supported by the National Natural Science Foundation of China (81000258, 81100436), the Natural Science Foundation of Jiangsu Province (BK2010586), the Bureau of Nanjing City Science and Technology Development Fund (201104014), the Open topic of State Key Laboratory of Reproductive Medicine (SKLRM-KF-201109) and the Nanjing Medical Science and Technique Development Foundation (QRX11210, QRX11211).

\section{References}

1. Barnes-Powell LL: Infants of diabetic mothers: the effects of hyperglycemia on the fetus and neonate. Neonatal Netw 26: 283-290, 2007.

2. Uzelac PS, Li X, Lin J, Neese LD, Lin L, Nakajima ST, Bohler H and Lei Z: Dysregulation of leptin and testosterone production and their receptor expression in the human placenta with gestational diabetes mellitus. Placenta 31: 581-588, 2010.

3. Ferrara A: Increasing prevalence of gestational diabetes mellitus: a public health perspective. Diabetes Care 30 (Suppl 2): S141-S146, 2007.

4. Lee AJ, Hiscock RJ, Wein P, et al: Gestational diabetes mellitus: clinical predictors and long-term risk of developing type 2 diabetes: a retrospective cohort study using survival analysis. Diabetes Care 30: 878-883, 2007.

5. Thadhani R, Powe CE, Tjoa ML, et al: First-trimester follistatin-like-3 levels in pregnancies complicated by subsequent gestational diabetes mellitus. Diabetes Care 33: 664-669, 2010.

6. Murphy VE, Smith R, Giles WB and Clifton VL: Endocrine regulation of human fetal growth: The role of the mother, placenta, and fetus. Endocr Rev 27: 141-169, 2006.

7. Luo SS, Ishibashi O, Ishikawa G, et al: Human villous trophoblasts express and secrete placental-specific microRNAs into maternal circulation via exosomes. Biol Reprod 81: 717-729, 2009.

8. Fu GD, Brkić J, Hayder H and Peng C: MicroRNAs in human placental development and pregnancy complications. Int J Mol Sci 14: 5519-5544, 2013.

9. Kotlabova K, Doucha J and Hromadnikova I: Placental-specific microRNA in maternal circulation - identification of appropriate pregnancy-associated microRNAs with diagnostic potential. J Reprod Immuno 89: 185-191, 2011.

10. Gauster M, Desoye G, Tötsch M and Hiden U: The placenta and gestational diabetes mellitus. Cure Diab Rep 12: 16-23, 2012.

11. Yilmaz-Aydogan H, Kurnaz O, Kucukhuseyin O, et al: Different effects of PPAR $\alpha$, PPARG and apoE SNPs on serum lipids in patients with coronary heart disease based on the presence of diabetes. Gene 523: 20-26, 2013.

12. Arck P, Toth B, Pestka A and Jeschke U: Nuclear receptors of the perocisome proliferator-activated receptor (PPAR) family in gestational diabetes: from animal models to clinical trials. Biol Reprod 83: 168-176, 2010.

13. Shi ZM, Wang J, Yan Z, et al: MiR-128 inhibits tumor growth and angiogenesis by targeting p70S6K1. PLoS One 7: e32709, 2012.

14. Morales-Prieto DM, Chaiwangyen W, Ospina-Prieto S, Schneider U, Herrmann J, Gruhn B and Markert UR: MicroRNA expression profiles of trophoblastic cells. Placenta 33: 725-734, 2012. 
15. Holdsworth-Carson SJ, Lim R, Mitton A, Whitehead C, Rice GE, Permezel M and Lappas M: Peroxisome proliferator-activated receptors are altered in pathologies of the human placenta: gestational diabetes mellitus, intrauterine growth restriction and preeclampsia. Placenta 31: 222-229, 2010.

16. Cresci S, Huss JM, Beitelshees AL, et al: A PPAR $\alpha$ promoter variant impairs ERR-dependent transactivation and decreases mortality after acute coronary ischemia in patients with diabetes. PLoS One 5: e12584, 2010.

17. Beaven SW and Tontonoz P: Nuclear receptors in lipid metabolism: targeting the heart of dyslipidemia. Annu Rev Med 57: 313-329, 2006.

18. Flavell DM, Pineda Torra I, Jamshidi Y, et al: variation in the PPAR $\alpha$ gene is associated with altered function in vitro and plasma lipid concentrations in Type II diabetic subjects. Diabetologia 43: 673-680, 2010.
19. Matsuda S, Kobayashi M and Kitagishi Y: Expression and function of PPARs in placenta. PPAR Res 2013: 256508, 2013.

20. Arck P, Toth B, Pestka A and Jeschke U: Nuclear receptors of the peroxisome proliferator-activated receptor (PPAR) family in gestational diabetes: from animal models to clinical trials. Biol Reprod 83: 168-176, 2010.

21. Dubé E, Ethier-Chiasson M and Lafond J: Modulation of cholesterol transport by insulin-treated gestational diabetes mellitus in human full-term placenta. Biol Reprod 88: 16, 2013. 